\title{
Evolução temporal da precipitação e atividade elétrica de uma tempestade com ocorrência de tempo severo
}

Time rush evolution and electric activity of a storm with severe weather event

\author{
Rebeca Fonseca de Oliveira Pereira ${ }^{1}$, Rachel Albrecht ${ }^{2}$ \\ ${ }^{1}$ Graduanda, Universidade Federal de Itajubá, Itajubá, Brasil \\ 2 Doutora, Universidade de São Paulo, São Paulo, Brasil
}

\begin{abstract}
Resumo
Este estudo teve como objetivo analisar a evolução espacial e temporal da precipitação e eletrificação de um Sistema Convectivo que ocorreu no dia 12 de março de 2012 sobre a cidade de São Paulo, durante o intervalo de tempo das 1830 às 1945 UTC. Foi feita uma análise do comportamento da estrutura tridimensional da refletividade de radar e das descargas elétricas do tipo Intra-Nuvem (IN) e Nuvem-Solo (NS) de duas redes de detecção de raios instaladas na região durante o Projeto CHUVA, afim de correlacionar a ocorrência de tempo severo com a evolução da corrente ascendente da nuvem, formação da precipitação e eletrificação desta tempestade. Observou-se pelas imagens de refletividade do Radar FCTH que, em alguns momentos, o SC apresentou valores altos de refletividade, sendo superiores a $70 \mathrm{dBZ} e$, indicando a presença de granizo dentro do sistema. Por meio do comportamento das descargas elétricas, observou-se também que estas acompanham o deslocamento e intensidade das correntes ascendentes $e$, por meio do lightning jump, é possivel detectar com antecedência se a tempestade está ou não se intensificando. Se sim, esta tempestade possivelmente pode vir se tornar um tempo severo e causar sérios danos a sociedade.

Palavras-chave: Sistema Convectivo, Radar, descargas elétricas, São Paulo.
\end{abstract}

\begin{abstract}
This study aimed to analyze the spatial and temporal evolution of rainfall and electrification of a Convective System, which occured on March 12, 2012 over the city of São Paulo, during the time interval from 1830 to 1945 UTC. An analysis was made of the behavior of the three dimensional structure of radar reflectivity and lightning type Intra-cloud (IN) and Cloud-Solo (NS), based on two lightning detection networks installed in the region during the RAIN Project, in order to correlate the occurrence of severe weather to the evolution of cloud ascending current, precipitation formation and electrification of this storm. It was observed by the reflectivity of the FCTH Radar images that, at times, the SC showed high values of reflectivity, with over $70 \mathrm{dBZ}$ and indicating the presence of hail within the system. Through the behavior of electrical discharges, it is also noted that these accompany the displacement and intensity of the updraft and through the lightning jump, it is possible to detect in advance whether if the storm is intensifying or not. If so, this storm could possibly become a severe weather and cause serious damage to society.
\end{abstract}

Keywords: Convective Systems, Radar, lightining, São Paulo. 


\section{Introdução}

Segundo o estudo de Gin e Guedes (2000) baseado em dados coletados em vinte e três estações meteorológicas, os autores concluíram que o período entre os meses de janeiro e março refere-se ao período com maior atividade de relâmpagos sobre o território brasileiro. Isso ocorre devido a forte atividade convectiva durante esta época, que geralmente está acompanhada a fatores como: instabilidades geradas por fenômenos de mesoescala e escala sinótica, aquecimento convectivo local, presença de zonas de convergência, entre outros. Os Sistemas Convectivos (SC), por sua vez, também são formados em condições atmosféricas semelhantes às condições citadas anteriormente. Esses sistemas tendem a se formar em locais com alto aquecimento radiativo, o que gera uma instabilidade condicional e/ou convectiva no perfil vertical e termodinâmico da atmosfera e, em locais que apresentem um forte cisalhamento vertical do vento, o que faz com que as correntes ascendentes e descendentes se apresentem de forma organizada, resultando em um SC mais severo e duradouro (SILVA DIAS, 1987).

Segundo Velasco e Fritsch (1987) e Vila (2008), os Sistemas Convectivos de Mesoescala (SCMs), são um dos principais responsáveis pelo excesso de precipitação na América do Sul durante as estações de primavera e verão. Porém, esses sistemas são reconhecidos não só pelo alto índice de precipitação, que algumas vezes pode resultar em alagamentos e desastres ambientais, como também pela alta atividade elétrica e forte rajada de vento, fatores estes que podem ser extremamente prejudiciais na rede de distribuição de energia elétrica (MACHADO et al., 2004). Segundo Willian et al. (1999), a definição de tempo severo é feita a partir de três limiares de determinadas características, que estão diretamente relacionadas com a intensidade das correntes ascendentes do sistema, como: (1) velocidade do vento, (2) tamanho das partículas de granizo e a (3) vorticidade do sistema. Para um sistema convectivo ser denominado de tempo severo, este deve apresentar pelo menos uma das três características citadas anteriormente. As correntes ascendentes, além de serem um dos principais responsáveis para formação de sistemas de tempo severo, também são responsáveis pela eletrificação e formação de relâmpagos no sistema. Isso ocorre, pois, essas correntes realizam o deslocamento de água super-resfriada no interior da nuvem, a qual é o agente principal para a formação de partículas de gelo, como: cristais de gelo, graupel e granizo, que por sua vez, são essenciais para a separação de carga elétrica no sistema.

A estrutura elétrica em uma tempestade pode ser definida como um modelo tripolo eletrostático (WILLIAMS, 1989), em que há três camadas de cargas: uma camada de distribuição de cargas negativas entre os níveis das isotermas de $-10^{\circ} \mathrm{C}$ e $-25^{\circ} \mathrm{C}$ e, duas camadas de distribuição de cargas positivas, que estão separadas pela camada negativa. Uma das teorias de formação do tripolo eletrostático é a teoria da precipitação, em que os elementos verticais e horizontais de precipitação dependem indiretamente da estrutura dinâmica da nuvem. Nessa teoria, o processo de precipitação se faz parcialmente responsável pela separação das cargas elétricas presentes no sistema, mecanismo esse que pode ser definido como não-indutivo (NI), isto é, não necessita de um campo elétrico prévio [e.g., REYNOLDS et al., 1957; BUSER e AUFDERMAUR, 1977; ILLIGWORTH e LATHAM, 1977; MARSHAL et al., 1978]. No processo NI, a separação das cargas elétricas pode ocorrer pela colisão entre as partículas de gelo em acresção (rimers) e as partículas de gelo menores. Essa colisão faz com que os rimers mais pesados passam a apresentar um sinal de carga e fiquem concentrados no meio da nuvem, enquanto os cristais de gelo passam a apresentar sinais opostos de cargas aos dos rimers e, por serem mais leves, são carregados pelas correntes ascendentes para os níveis mais altos do sistema, resultando em uma separação de partículas por tamanhos e sinais de cargas.

Assim, no dia 12 de março de 2012, foi observado um intenso Sistema Convectivo (SC) atuando sobre a cidade de São Paulo. De acordo com o Boletim Meteorológico gerado pelo Centro de Previsão de Tempo e Estudos Climáticos (CPTEC), as cartas sinóticas analisadas para esse dia indicaram a presença de um cavado em altos níveis na região, o qual apresentou divergência 
em seu nível de atuação e convergência em baixos níveis. Esse sistema, associado com a instabilidade termodinâmica da região, fez com que a instabilidade e a convergência de umidade prevalecessem sobre a cidade de São Paulo, induzindo uma forte atividade convectiva. Segundo o Relatório de Chuvas da Prefeitura de São Paulo, o regime de precipitação desse sistema iniciou-se aproximadamente às 15h45m, perdurando até às $17 \mathrm{~h} 20 \mathrm{~m}$ em algumas regiões da cidade. Durante o período de precipitação, várias regiões de São Paulo receberam notificações de Estado de Atenção, registrando valores de precipitação acumulada de até 42,0 $\mathrm{mm}$ durante o dia. Além de um forte regime de precipitação, o SC também causou queda de granizo e fortes rajadas de vento. Durante o período analisado, das 1830 às 1945 UTC, segundo o Centro de Gerenciamento de Emergências (CGE) da cidade de São Paulo, foram registradas quedas de seis árvores localizadas em diferentes regiões e, segundo informações da mídia, foram registrados cinco locais com ocorrência de queda de granizo e nove locais com registros de alagamento.

O objetivo desse estudo foi analisar a evolução espacial e temporal da precipitação e eletrificação deste SC. Para tanto, foi feita uma análise do comportamento da estrutura tridimensional da refletividade de radar e das descargas elétricas do tipo Intra-Nuvem (IN) e Nuvem-Solo (NS) de duas redes de detecção de raios instaladas na região durante o Projeto CHUVA, afim de correlacionar a ocorrência de tempo severo com a evolução da corrente ascendente da nuvem, formação da precipitação e eletrificação desta tempestade.

\section{Metodologia}

O monitoramento do sistema estudado foi feito por meio do Radar Meteorológico de Ponte Nova, operado pela Fundação Centro Tecnológico de Hidráulica (FCTH), com imagens disponíveis continuamente em um intervalo de cinco minutos sobre a cidade de São Paulo. Além disso, para os dados de descargas atmosféricas, foram utilizados dois sistemas de localizações de relâmpagos instalados nesta região durante o Projeto CHUVA (Machado et al., 2014), o Lightning Mapping Array (LMA) e o Lightning
Location Network (LINET). O SC que causou diversos eventos de tempo severo foi rastreado manualmente através da evolução temporal da refletividade nos campos de CAPPI em $3 \mathrm{~km}$ de altura. Nesse rastreamento foi determinada uma região retangular em torno do sistema, na qual cortes verticais da tempestade foram realizados. Com a junção dos dados de refletividade e raios, foi possível reproduzir o deslocamento do sistema, gerando gráficos de latitude por longitude, latitude por altura e longitude por altura, totalizando em perfis horizontais e verticais do sistema durante o período analisado.

\section{Resultados}

Com os produtos CAPPI do radar FCTH disponibilizados a cada cinco minutos, foi possível reproduzir dezesseis campos de refletividade durante o período de atuação do SC na cidade de São Paulo, ou seja, das 1830 às 1945 UTC. Com os dados dos sensores de raios disponibilizados pelo Projeto CHUVA, também foi possível analisar o comportamento bidimensional e tridimensional das descargas elétricas presentes no sistema.

\subsection{Acompanhamento Bidimensional:}

$\mathrm{Na}$ Figura 1 é possível realizar o acompanhamento do SC indicado pelo retângulo em cada intervalo de tempo. É possível localizar também seu núcleo convectivo de maior severidade, indicado pelo encontro entre a linha horizontal com a vertical, e também pelo índice de maior refletividade. Nas primeiras imagens [Figura $1(a ; b ; c)$ ] nota-se que o SC começa a adentrar a cidade de São Paulo com uma área de extensão de cerca de $380 \mathrm{~km}^{2}$ (>30 dBZ) e com um núcleo convectivo intenso, com índices de refletividade acima de $70 \mathrm{dBZ}$. Ao analisar as imagens consecutivas, é possível observar que o SC se expande e, ao final do seu ciclo de vida, este apresenta uma extensão de aproximadamente $855 \mathrm{~km}^{2}$. Nas imagens seguintes [Figura 1 (d;e;f;g;h)], o SC amplia sua área de extensão, comparada com as primeiras imagens, alcançando um tamanho de cerca de $547 \mathrm{~km}^{2}$. É possível notar também que a área de seu núcleo convectivo também se expande, porém, perde um pouco de sua intensidade, 
registrando valores de refletividade abaixo de 60 dBZ. Porém, é nesse intervalo de tempo que algumas regiões de São Paulo registraram casos de alagamentos e quedas de árvores, indicando que, apesar do sistema aparentemente ter diminuído sua intensidade, seu potencial de precipitação e rajadas de vento se apresentaram intensos o suficiente para causar danos à sociedade.

Já nas imagens subsequentes [Figura 1 (i;j;k;l)], um dos focos principais desse estudo, nota-se pelos índices de refletividade maiores que $55 \mathrm{dBZ}$ que, o núcleo convectivo do SC diminui seu tamanho, isto é, se contrai e registra uma área de aproximadamente $95 \mathrm{~km}^{2}$, aumentando sua intensidade e consequentemente seu índice de refletividade. É nesse intervalo de tempo que casos de granizos são registrados na Zona Norte de São Paulo e que o SC registra o maior valor de refletividade, de aproximadamente $73 \mathrm{dBZ}$. Nas últimas imagens [Figuras 1 (m;n;o;p)], o núcleo convectivo antes registrado como de maior intensidade começa a se dissipar, apresentando um índice de refletividade abaixo de $55 \mathrm{dBZ}$. Na região leste do sistema, outro núcleo é formado e se intensifica, registrando índices de refletividade superiores a $70 \mathrm{dBZ}$ e causando alagamento na Zona Leste de São Paulo, registrado pelo CGE.

Nas imagens da Figura 1, observa-se também o comportamento das descargas elétricas de acordo com o ciclo de vida e o deslocamento do SC. Nota-se que, há uma maior densidade de atividade elétrica (indicado pelos pontos em cinza) no núcleo convectivo do SC, pois, é nessa região que estão localizadas as correntes ascendentes de maior intensidade no SC e, como observado por Workman e Reynolds (1949) e Schultz et al (2009), a presença de um raio está intimamente ligada com a intensidade das correntes ascendentes existentes no sistema considerado tempo severo, pois, é nessa região onde há uma maior quantidade de grandes hidrometeoros de gelo e quanto maior a intensidade da corrente ascendente mais colisões haverão e mais cargas elétricas serão transferidas. Assim, como foi possível observar o deslocamento do núcleo convectivo, também é possível observar o descolamento das descargas elétricas, que sempre acompanham a região do SC de maior intensidade. Nas imagens da Figura
1 (i;j;k;l), período em que foi registrado queda de granizo na Zona Norte de São Paulo, observa-se que nas duas primeiras imagens [Figuras $1(\mathrm{i} ; \mathrm{j})$ ] as descargas elétricas estão intensamente concentradas na região do núcleo convectivo do $\mathrm{SC}$, indicando um grande número de raios IN e NS, isto é, confirmando a presença de granizo no interior do sistema. Nota-se ainda que, principalmente nas imagens da Figura 1 (n;p), a medida que o SC começa a se dissipar e as correntes ascendentes perdem intensidade, a densidade de descargas elétricas também diminui e, começam a se espalhar para a região estratiforme do sistema. Essa região pode ser localizada no SC pelos índices de refletividade com valores entre 40 à $55 \mathrm{dBZ}$ e, com a presença da banda brilhante, caracterizada pela precipitação estratiforme. Analisando o comportamento dos raios Intra-Nuvem (IN pontos pretos) e Nuvem-Solo (NS - pontos verdes), também é possível observar que os mesmos acompanham o deslocamento do núcleo convectivo do SC, pois é nessa região que se encontram as correntes ascendentes de maior intensidade, assim como observado por William et al. (1999), em que os autores chegaram à conclusão que as correntes ascendentes aparentam ser um dos responsáveis para a origem das descargas elétricas IN e NS.

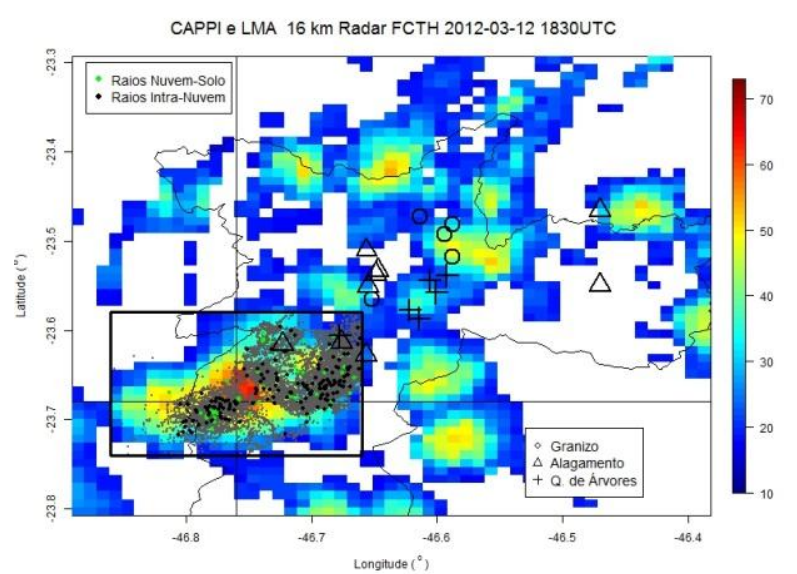

(a)

Figura 1 - Campos de refletividade (dBZ) gerados pelo radar FCTH do dia 12 de março de 2012, no intervalo das 1830 às 1945 UTC sobrepostos com dados dos sensores de raios LMA e LINET. 


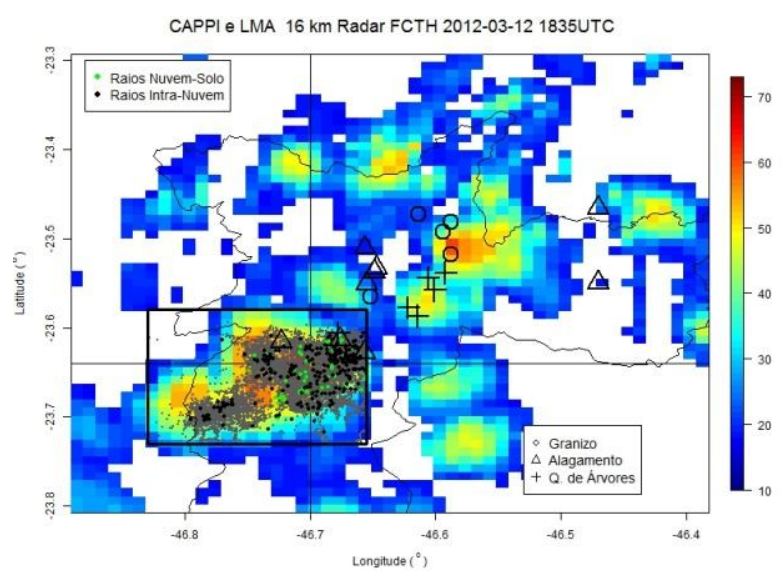

(b)

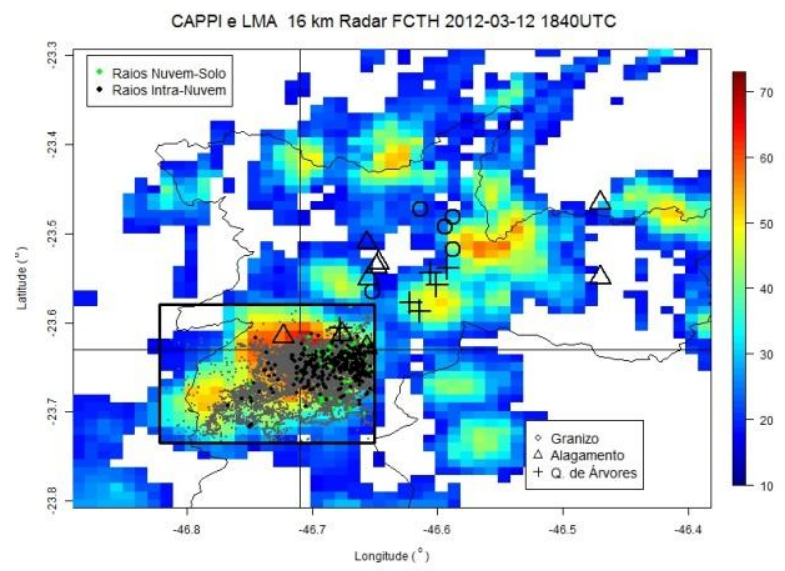

(c)

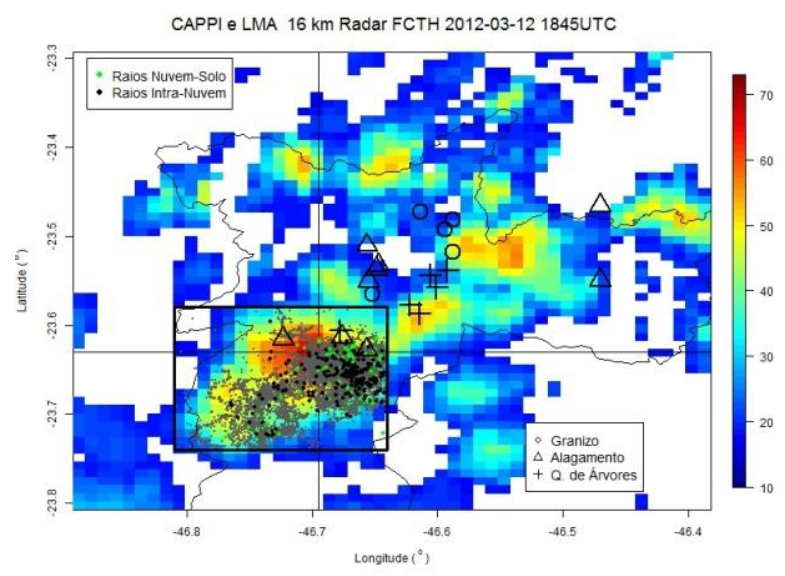

(d)

(Continuação da Figura 1.)

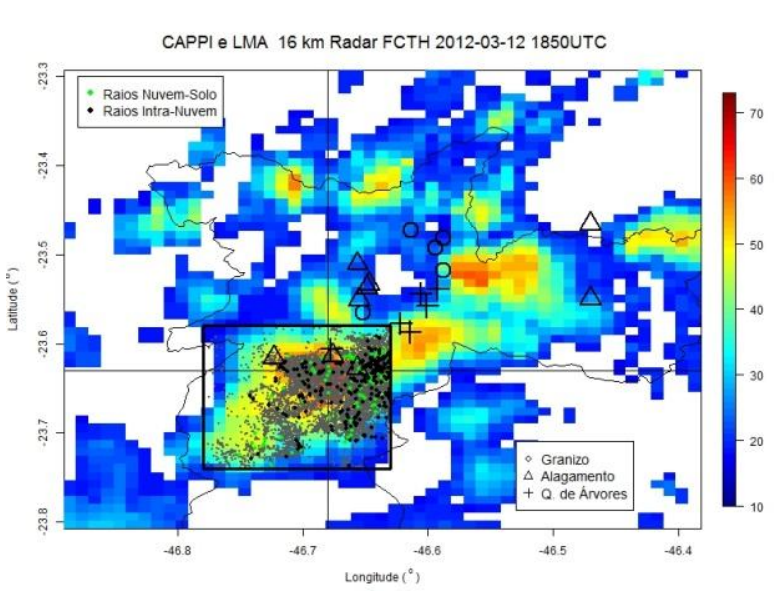

(e)

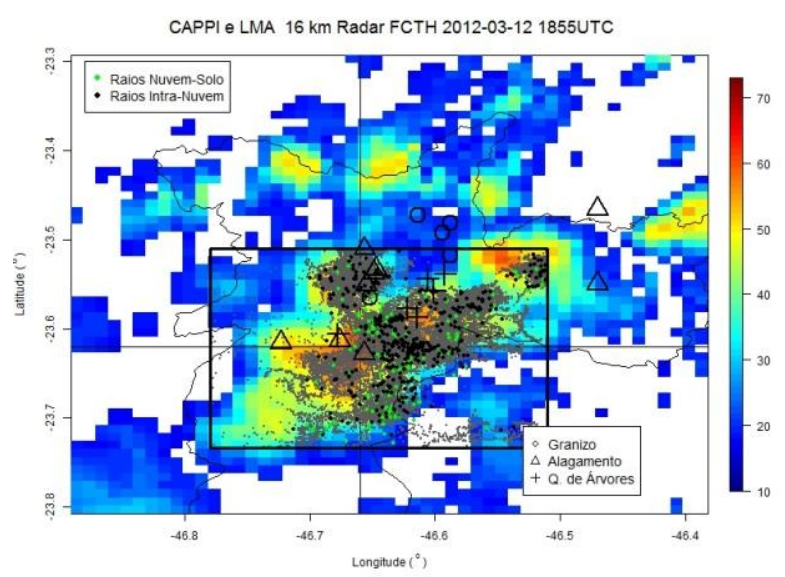

(f)

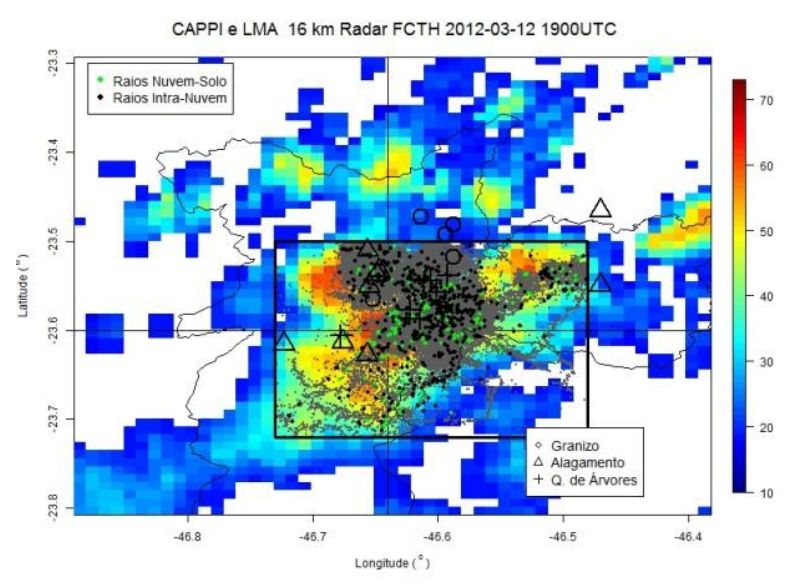

(g)

(Continuação da Figura 1.) 


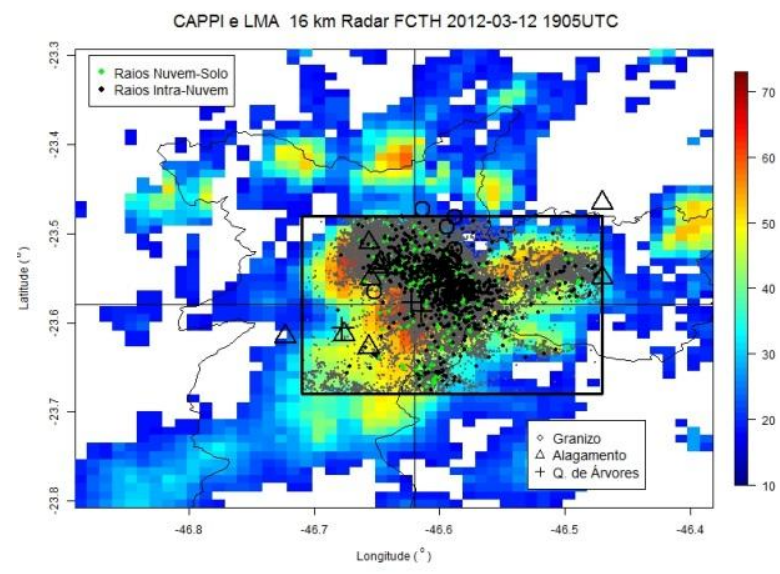

(h)

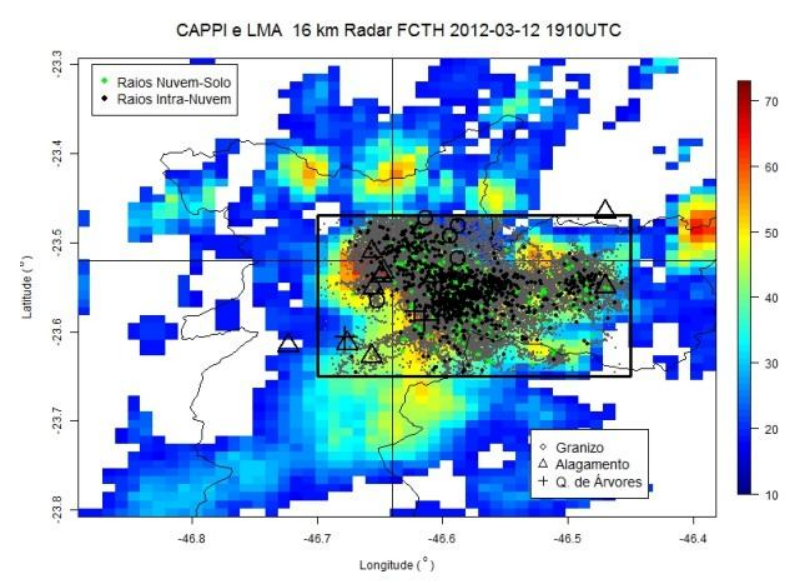

(i)

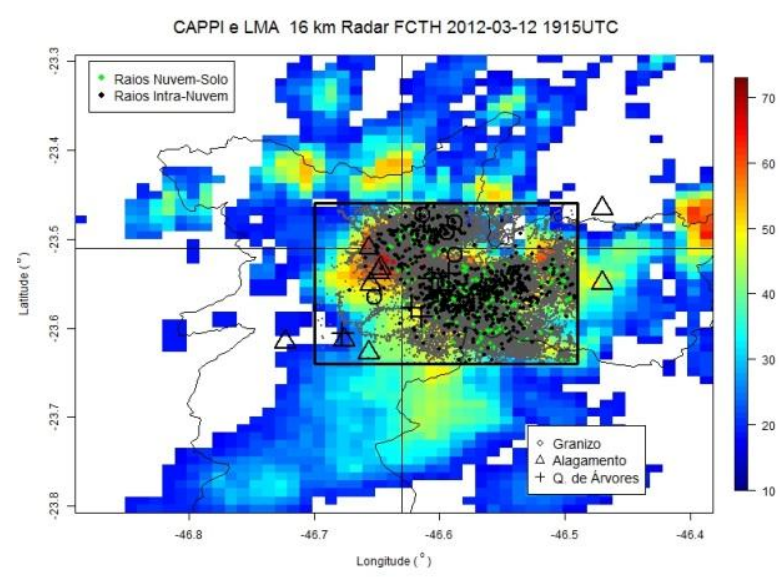

(j)

(Continuação da Figura 1.)

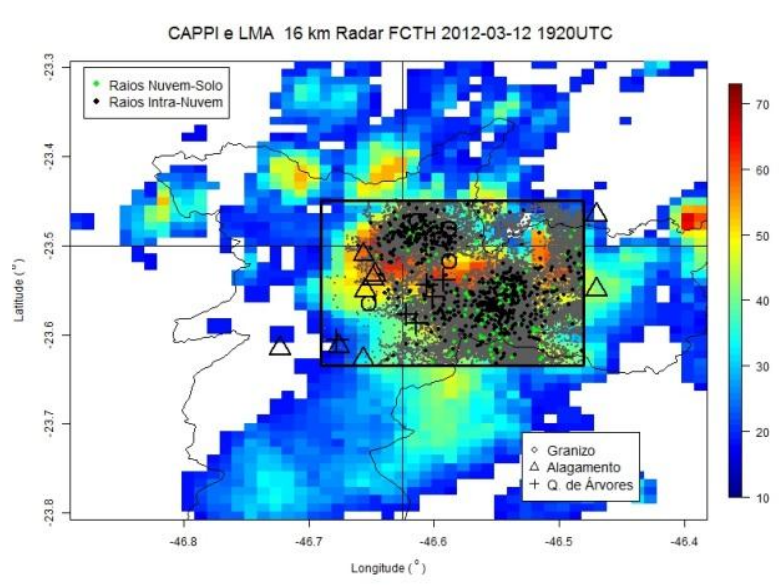

(k)

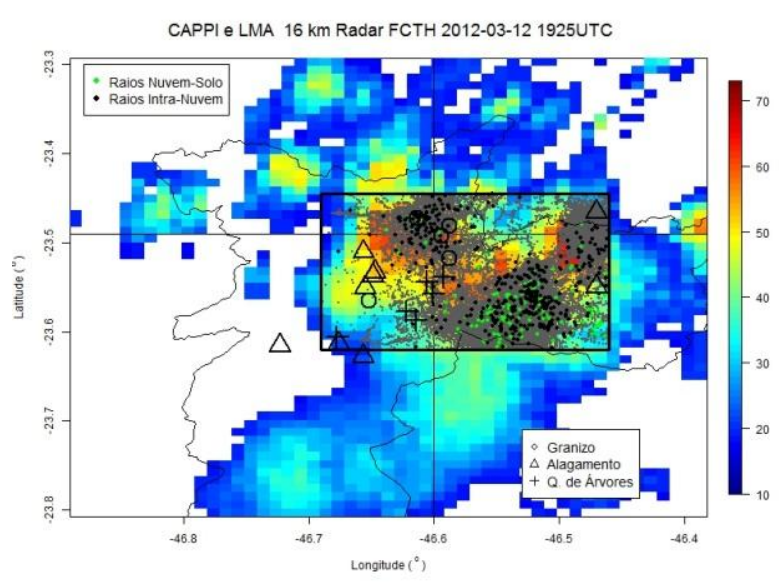

(1)

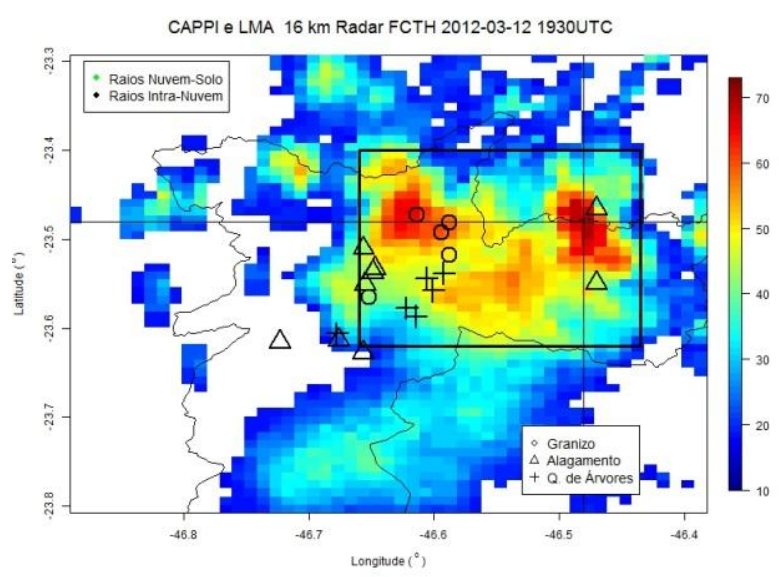

(m)

(Continuação da Figura 1.) 


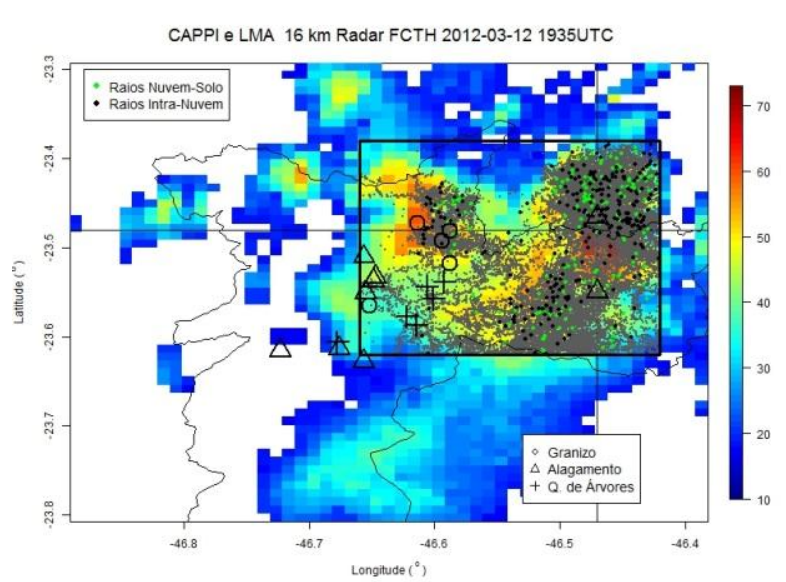

(n)

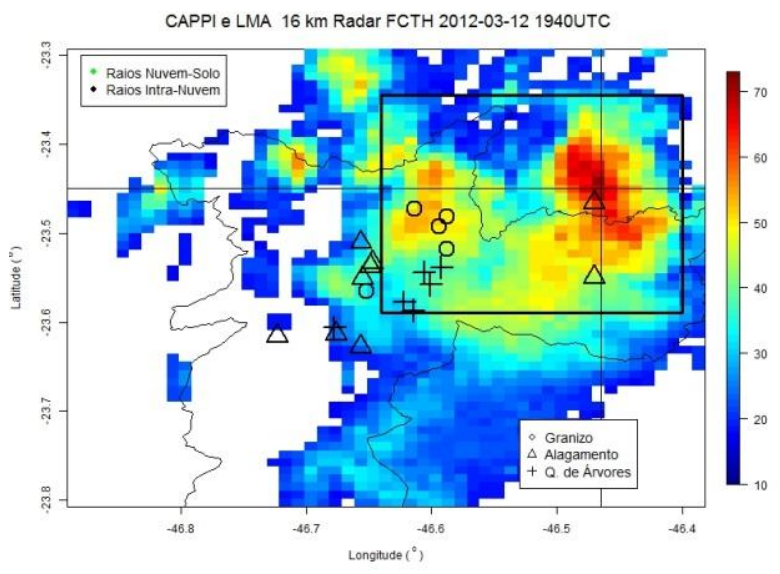

(o)

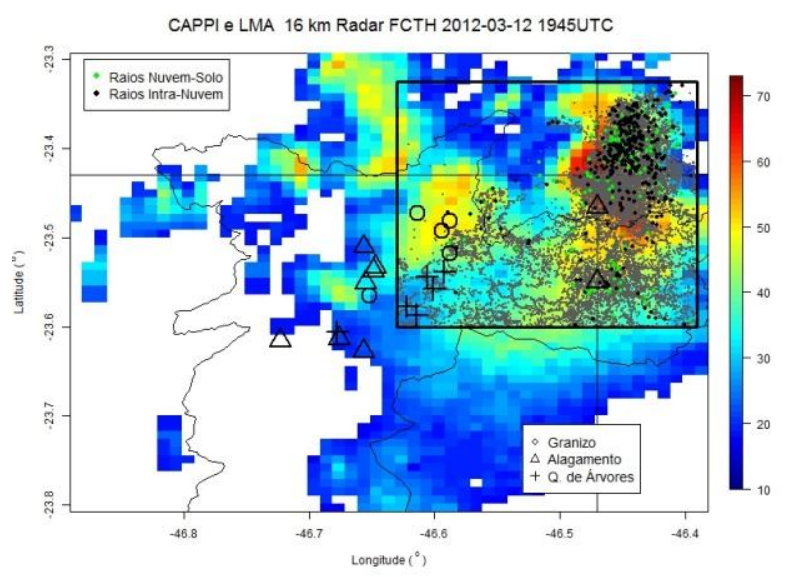

(p)

(Continuação da Figura 1.)

\subsection{Corte Latitudinal - Acompanhamento Vertical:}

Com o perfil vertical do SC é possível não só visualizar as características internas do sistema, como também analisar sua extensão vertical e horizontal. O corte latitudinal foi realizado no ponto em que o SC apresentava o maior valor de refletividade de cada campo de refletividade em
$3 \mathrm{~km}$ de altura, ou seja, no ponto em que as linhas horizontal e vertical da Figura 1 se cruzavam. Na Figura 2, é possível observar que o sistema alcançou os altos níveis da troposfera, pois, em todas as imagens, o SC apresenta um índice de refletividade de no mínimo $10 \mathrm{dBZ}$ acima de $14 \mathrm{~km}$ de altura, sendo este o limiar mínimo de presença de água precipitável em um sistema. Uma vez que a coluna atmosférica é superior, a formação de chuva pode ser favorecida pelo maior tempo na geração de grandes hidrometeoros que, como citado por Reynolds et al (1957), Buser e Aufdermaur (1977) entre outros autores, são essenciais para a formação de cargas elétricas no interior da nuvem por meio do processo de colisão entre si.

Nas primeiras quatro imagens [Figura 2 (a;b;c;d)] é possível observar que o sistema está se intensificando pois, seu núcleo convectivo apresenta um crescimento nos valores de refletividade, de $55 \mathrm{dBZ}$, para valores acima de $70 \mathrm{dBZ}$ durante este intervalo de tempo. Nas imagens seguintes [Figura 2 (e;f;g;h)] nota-se que o núcleo convectivo mantem uma refletividade praticamente constante, com valores de aproximadamente $60 \mathrm{dBZ}$. Porém, nas imagens correspondentes ao registro de queda de granizo em superfície [Figura $2(\mathrm{i} ; j ; \mathrm{k} ; \mathrm{l})]$ observa-se que o núcleo convectivo volta a se intensificar, registrando índices de refletividade acima de 70 dBZ, comprovando a queda de granizo em superfície durante o intervalo das 1910 às 1925 UTC (isto é, das $16 \mathrm{~h} 10 \mathrm{~m}$ às $16 \mathrm{~h} 25 \mathrm{~m}$ hora local), como foi observado pelos moradores da região. Nas últimas imagens [Figura 2 (m;n;o;p)] nota-se que o primeiro núcleo convectivo se dissipa gradativamente, registrando valores de refletividade abaixo de $60 \mathrm{dBZ}$, enquanto outro núcleo convectivo atinge seu estágio de maturação e registra um índice de refletividade acima de $70 \mathrm{dBZ}$, provocando a propagação e renovação do SC.

Como citado, a estrutura elétrica de uma tempestade é um tripolo eletrostático, em que uma camada de cargas negativas se espalha entre as isotermas de $-10^{\circ} \mathrm{C}$ e $-25^{\circ} \mathrm{C}$ (região de formação e presença de graupel e granizo) e, duas camadas de cargas positivas são divididas ao meio pela distribuição de carga negativa, formando um modelo de tripolo. Nas imagens da Figura 2, é possível observar claramente esse modelo de tripolo eletromagnético. A 
propagação das cargas elétricas dos raios (indicados pelos pontos cinza) ocorrem devido ao rompimento das regiões de separação entre as cargas negativas e positivas que, em consequência disso, para que retornem ao equilíbrio interno do sistema, as cargas negativas são transportadas através de correntes elétricas para a região contendo cargas positivas e viceversa. Se as cargas foram transportadas somente dentro da nuvem são denominados $\mathrm{IN}$, e se transportaram cargas para o solo são denominados NS. A rede LINET fornece a localização da descarga de retorno nesses raios também representados na Figura 2 (pontos pretos - IN, pontos verdes - NS).

Observa-se na Figura 2 que, as descargas elétricas indicadas pelos pontos em cinza estão localizadas mais intensamente nas regiões de distribuição de cargas positivas (níveis de $5 \mathrm{~km}$ e $10 \mathrm{~km}$ de altura). Isso não implica que na região central do modelo de tripolo eletrostático, isto é, na região de cargas negativas, não estejam ocorrendo as descargas elétricas. A falta de registro de descargas elétricas pelos sensores LMA nessa região pode ser explicada pelo fato de que neste local há o deslocamento dos líderes positivos da ramificação das descargas, que não são captados pelos sensores. Já na região da base e do topo do tripolo, regiões de cargas positivas, há o deslocamento dos líderes negativos que são captados pelos sensores. Quando esses líderes encontram as regiões de concentração de cargas positivas e negativas, a descarga elétrica de retorno ocorre a fim de neutralizar esses centros.

Analisando detalhadamente cada imagem, nota-se que, como observado anteriormente, as descargas elétricas tendem a se localizar nas regiões onde se encontram as correntes ascendentes de maior intensidade e onde há maiores quantidades de hidrometeoros, ou seja, nos núcleos convectivos. Nas primeiras imagens [Figura 2 (a;b;c;d;e)] observa-se a estrutura tripolar e, que os raios IN estão espalhados por todo o núcleo convectivo. Os raios NS próximos à superfície também seguem o mesmo comportamento, acompanhando as regiões de maior refletividade. No intervalo das imagens (f;g;h) da Figura 2 observa-se um grande aumento repentino do número de descargas elétricas, o que é denominado de Lightning Jump. Com o lightning jump, é possível prever o desenvolvimento do sistema, já que esse aumento drástico do número de descargas elétricas indica que as correntes ascendentes do sistema estejam se intensificando e, posteriormente, a tempestade poderá se tornar um tempo severo. A ocorrência do tempo severo pode ser comprovada nas imagens seguintes, correspondentes ao horário da queda de granizo [Figura 2 (i;j;k;l)] observado em superfície. Notase neste intervalo de tempo que a densidade de descargas elétricas continua alta, acompanhando o aumento da severidade do sistema. Porém, a partir da imagem correspondente ao horário das 1930 UTC [Figura 2 (m)], o sistema começa a se desintensificar e, consequentemente, as descargas elétricas começam a se dissipar e a se espalharem para a região estratiforme do SC.

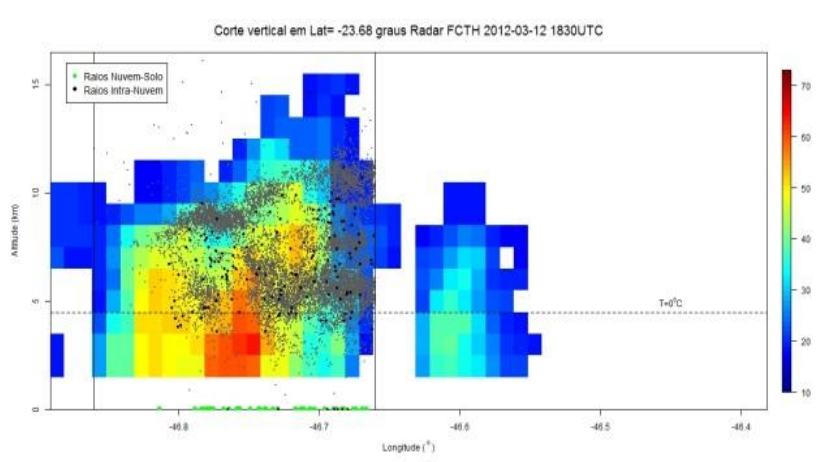

(a)

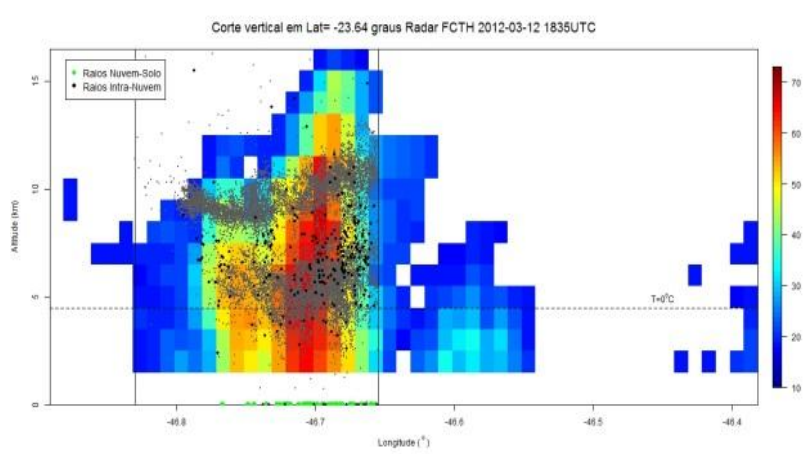

(b)

Figura 2: Corte latitudinal dos campos de refletividade (dBZ) gerados pelo radar FCTH do dia 12 de março de 2012, no intervalo das 1830 às 1945 UTC; sobrepostos com dados dos sensores de raios LMA e LINET. 


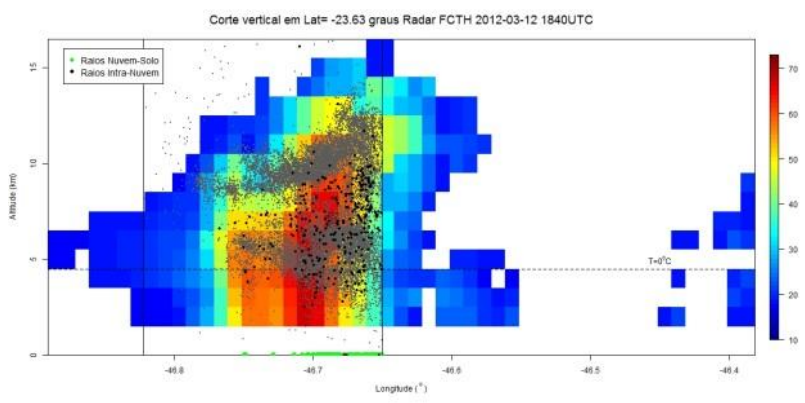

(c)

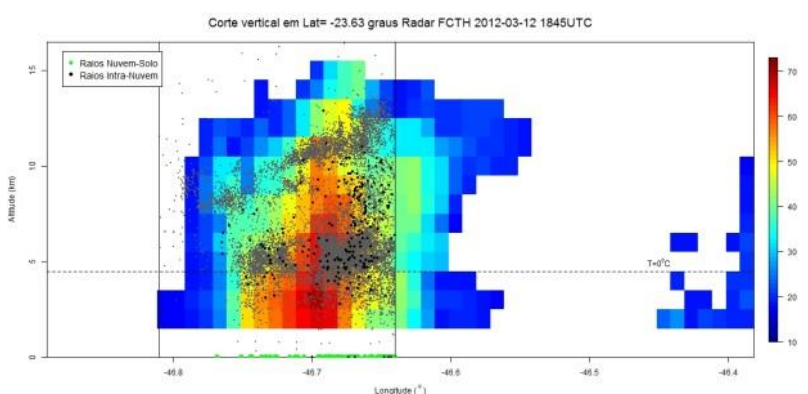

(d)

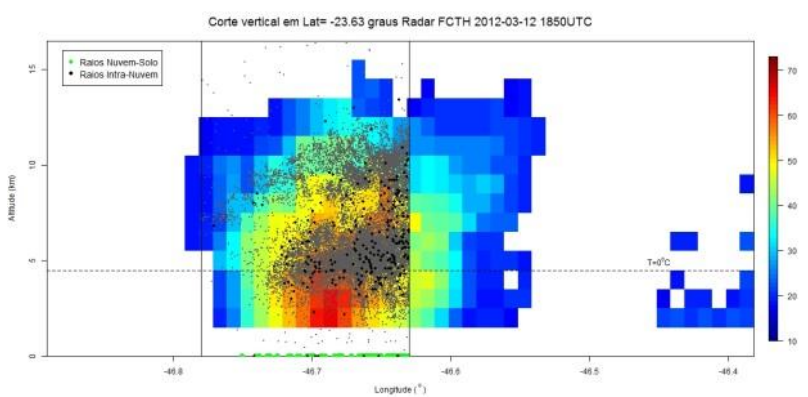

(e)

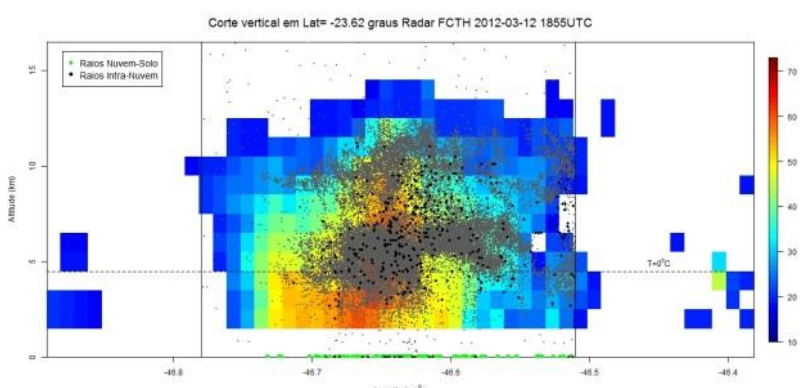

(f)

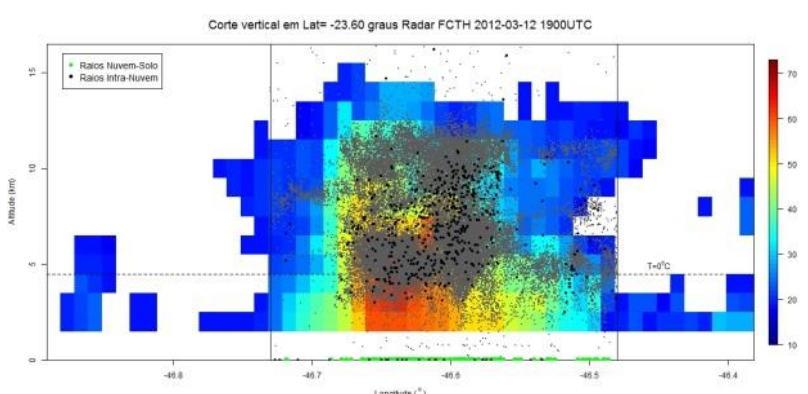

(g)

(Continuação da Figura 2).

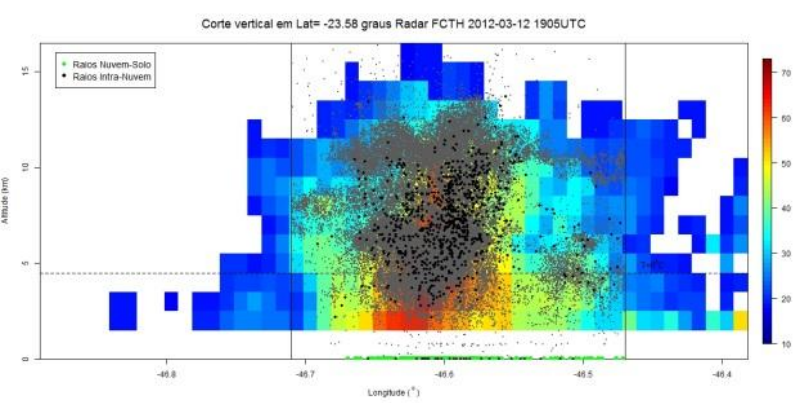

(h)

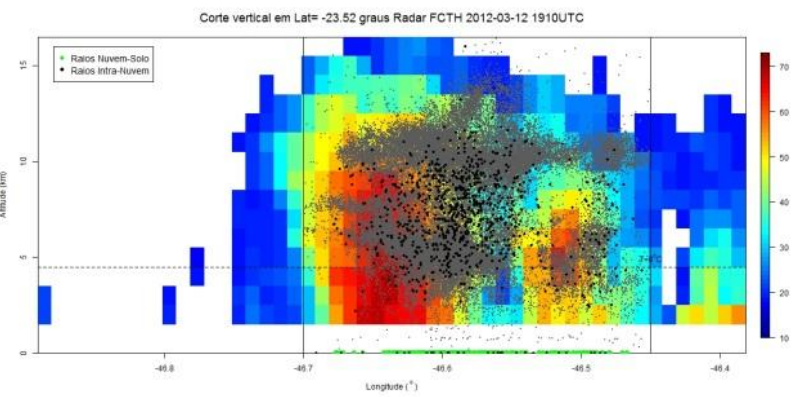

(i)

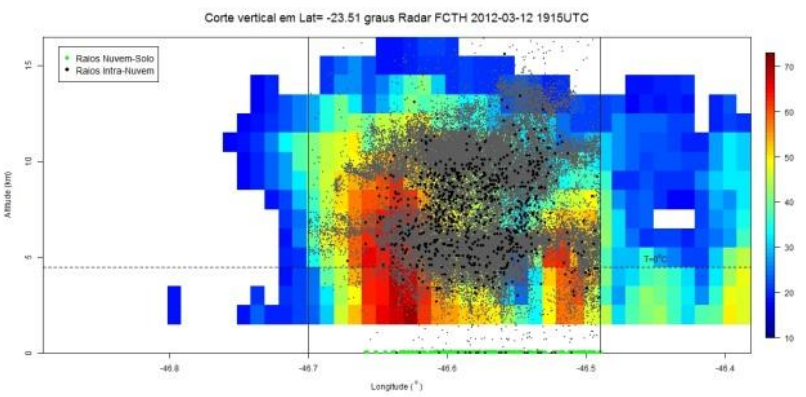

(j)

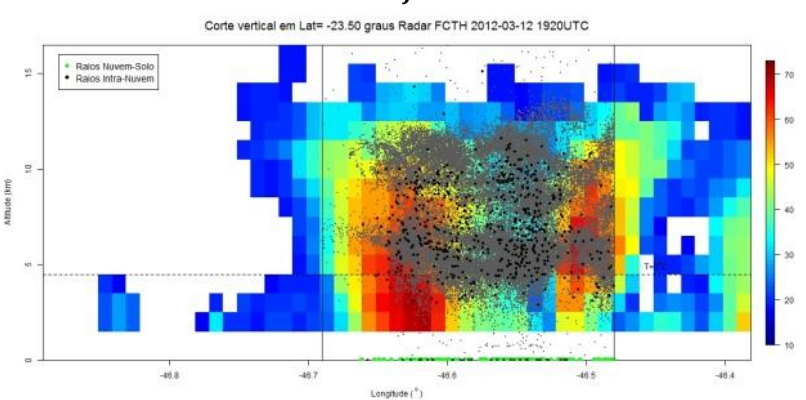

(k)

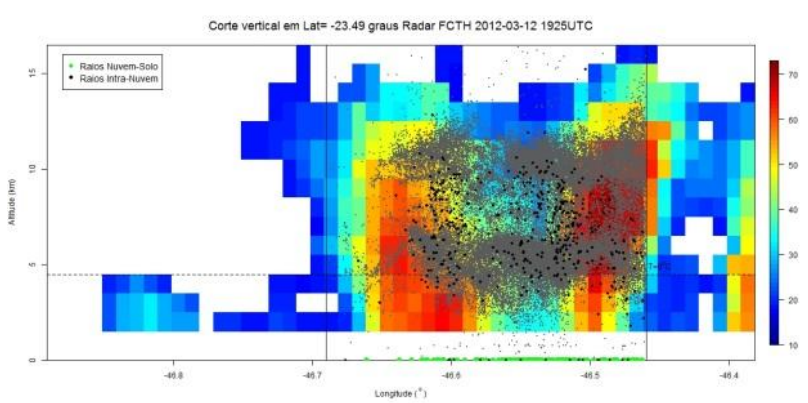

(1)

(Continuação da Figura 2). 


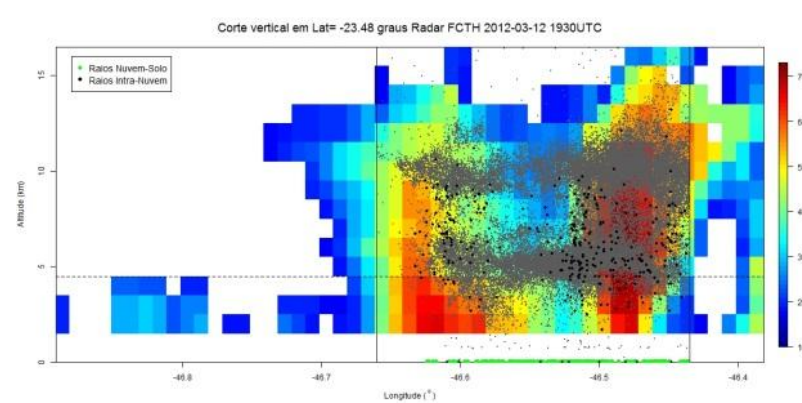

(m)

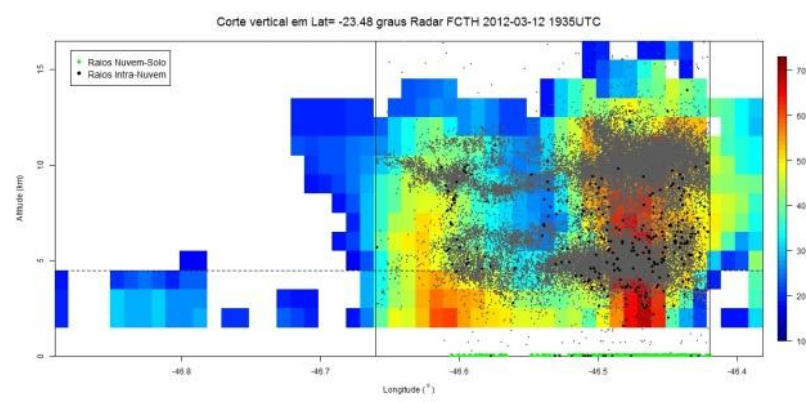

(n)

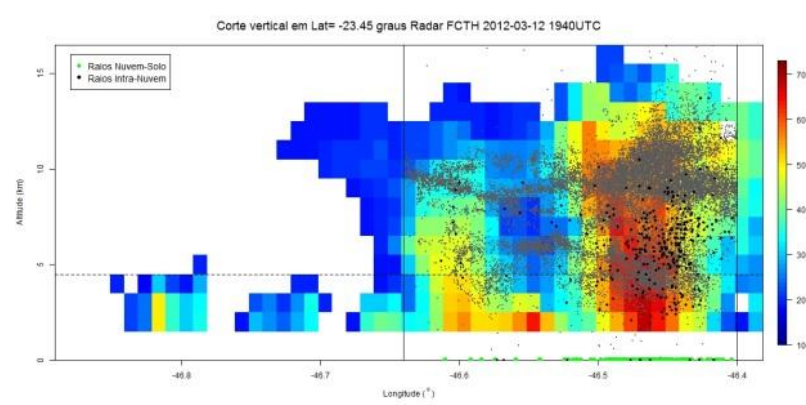

(o)

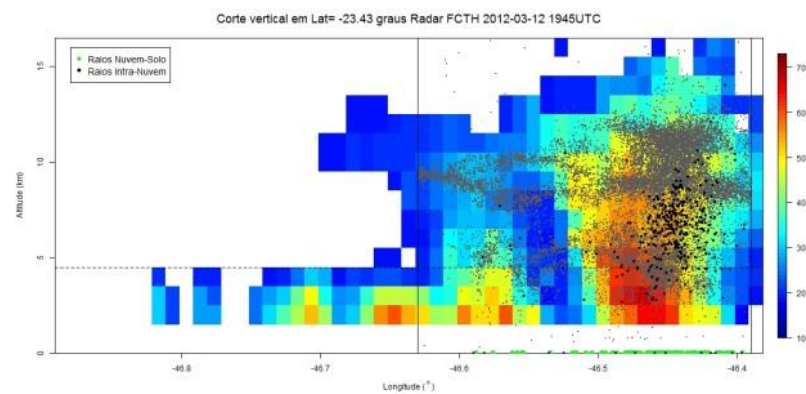

(p)

(Continuação da Figura 2).

\section{Conclusões}

Após feita a análise temporal e espacial da evolução da precipitação e eletrificação do SC estudado, conclui-se que este sistema possivelmente apresentou seus três estágios de ciclo de vida (estágio inicial, estágio de maturação e estágio de dissipação) sobre a cidade de São Paulo durante o intervalo de tempo estudado. É possível observar essas características por meio do comportamento dos índices de refletividade fornecidos pelo Radar FCTH e pelo tamanho do sistema em si e de seu núcleo convectivo, que podem definir se o sistema está se intensificando ou desintensificando. Outra característica importante neste estudo foram as descargas elétricas, que por meio do lightning jump, foi possível concluir que a tempestade se intensificou através de suas correntes ascendentes e, posteriormente, gerou tempo severo. A ocorrência de tempo severo veio a se confirmar com o registro de queda de granizo em superfície, observado por moradores da cidade de São Paulo, obedecendo pelo menos duas condições impostas por Willian et al. (1999) citadas anteriormente para que um sistema seja considerado um tempo severo. E, com os dados de radar sobrepostos com os dados dos sensores de descargas elétricas, conclui-se que se houvesse um monitoramento contínuo da tempestade estudada em tempo real, com a observação do lightning jump, provavelmente seria possível prever a queda de granizo nos minutos subsequentes, podendo evitar alguns danos à sociedade.

Por fim, assim como Deierling et al. (2008), a relação entre as descargas elétricas e as tempestades se faz de tal importância que, quando essa relação qualitativa é definida, os parâmetros encontrados podem ser utilizados para melhorar outras aplicações, como: melhoria das previsões dos modelos de mesoescala por meio das informações de variáveis convectivas, melhoria da definição do papel da convecção no clima global, melhorias na quantidade de precipitação estimada e melhoria na previsão à curto prazo (nowcasting) da evolução e dissipação da tempestade. Definir esses fatores com maior precisão e objetividade não só trará melhorias para o meio científico, como também para a sociedade, que estará melhor preparada para enfrentar eventos extremos, além de aumentar sua confiança perante os serviços prestados pelos meteorologistas.

\section{Referências}

BUSER, O., AUFDERMAUR, A. N., Electrification by collisions of ice particles on ice or metal targets, In Electrical Processes in 
Atmospheres. Eds. H. Dolezalek and R. Reiter

Darmstadt, Germany, 294, 1977;

DEIERLING, W., PETERSEN, W.A., LATHAM, J., ELLIS, S. e CHRISTIAN, H.J.: The Relationship Between Lightning Activity and Ice Fluxes in Thunderstorms. Journal of Geophysical Research, Vol. 113, 2008;

GIN, R.B.B, GUEDES, R.L. Climatologia de Relâmpagos no Brasil: Análise Preliminar. Congresso Brasileiro de Meteorologia, 11, 2000;

ILLIGWORTH, A. J., LATHAM J., Calculations of electric field growth, field structure, and charge distributions in thunderstorms, Quart. J. Royal Meteor. Soc., vol. 103, p. 277, 1977;

MACHADO, L.A., VILA, D. e MACEDO, S.R., Forecast and Tracking of Active Convective Cells - Guia do Usuário. Instituto Nacional de Pesquisas Espacias (INPE); 2004;

MARSHAL, B. J. P., LATHAM, J., SAUNDERS, C. P. R., A laboratory study of charge transfer accompanying the collision of ice crystals with a simulated hailstone, Quart. J. Royal Meteor. Soc., vol. 104, p. 163, 1978;

REYNOLDS, S. E., BROOK, M., GOURLEY, M. F., Thuderstorm charge separation, J. Meteor., vol. 14, p. 426, 1957;

VELASCO, I. e FRITSCH, J. M., Mesoscale convective complexes in the Americas. $J$. Geophys. Res. 92, 9591-9613, 1987;

VILA, D., L. A. T. MACHADO, H. LAURENT e I. VELASCO: Forecast and Tracking the Evolution of Cloud Clusters (ForTraCC) Using Satellite Infrared Imagery: Methodology and Validation. Weather and Forecasting, v. 23, n. 2, PP. 233-245, 2008;

WILLIAMS, E., The tripole structure of thunderstorms, J. Geophys. Res., vol. 94, p.13151; 1989; 